\title{
Research on Electrostatic Monitoring of Tribo-Contacts with Dynamic Adaptive Fusion Method
}

\author{
Ruochen Liu $\mathbb{D}^{1,},{ }^{1,2}$ Han Wang $\mathbb{D}^{2,3}$ Jinwu Zhang $\mathbb{C}^{1},{ }^{1}$ Shuangshuang $\mathbf{G u} \mathbb{D}^{1}{ }^{1}$ \\ and Jianzhong Sun $\mathbb{D D}^{3}$ \\ ${ }^{1}$ School of Automobile and Traffic Engineering, Jiangsu University of Technology, Changzhou 213001, China \\ ${ }^{2}$ Center for Advanced Life Cycle Engineering (CALCE), University of Maryland, College Park, MD 20742, USA \\ ${ }^{3}$ College of Civil Aviation, Nanjing University of Aeronautics and Astronautics, Nanjing 211106, China
}

Correspondence should be addressed to Ruochen Liu; liuruochen_nuaa@163.com

Received 10 August 2021; Revised 27 November 2021; Accepted 2 December 2021; Published 4 January 2022

Academic Editor: Ricardo Escobar

Copyright (C) 2022 Ruochen Liu et al. This is an open access article distributed under the Creative Commons Attribution License, which permits unrestricted use, distribution, and reproduction in any medium, provided the original work is properly cited.

Electrostatic monitoring is a unique and rapid developing technique applied in the prognostics and health management of the tribological system based on electrostatic charging and sensing phenomenon. It has considerable advantages in condition monitoring of tribo-contacts with high sensitivity and resolution. Unfortunately, the monitoring result can be affected due to the switch of operating conditions that reduces its accuracy. This paper presents a dynamic adaptive fusion approach, moving window local outlier factor based on electrostatic features to overcome the influence. Life cycle experiments of rolling bearings and railcar gearbox were carried out on an electrostatic monitoring platform. The MWLOF method was used to extract and analyze the experimental data, combined with the Pauta criterion to judge wear faults quantitatively, and compare with other feature extraction results. It is verified that the proposed method can overcome the influence of changes in working conditions on the monitoring results, improve the monitoring sensitivity, and provide an accurate reference for friction and wear faults.

\section{Introduction}

Online condition monitoring is a significant concern in improvements of prognostics and health management (PHM) for mechanical equipment [1-3]. Necessary actions can be taken with scheduled maintenance to avoid the failure of mechanical equipment with successful condition monitoring techniques [4-7]. Nowadays, a unique developing technique that is called the electrostatic monitoring method has been investigated based on electrostatic charging phenomena $[8,9]$. This technique has been proved to be effective for tribological contacts by using electrostatic sensors [10-13].

The electrostatic monitoring technique was initially used to detect wear debris of components deterioration presented in the exhaust path of gas turbine engines [14-16]. Later, it is introduced to apply in the friction and wear of mechanical systems with researches on the monitoring of sliding rolling and bearing wear systems [17-20]. After a series of experimental works, the electrostatic monitoring technique has been proved to be effective with its high sensitivity in condition monitoring of tribo-contacts [21-24]. Unlike conventional monitoring methods such as temperature and vibration that measure the secondary effects of wear, electrostatic monitoring is a direct measurement with detected electrostatic charges to the wear components by using electrostatic sensors [25-27]. It can provide not only the detection of severe failures but also early warnings and reflect the degradation of mechanical systems.

Although many researches have been conducted on the electrostatic monitoring technique, it is still challenging because the electrostatic monitoring signals can be influenced by the change of operating conditions. Current experiments of electrostatic monitoring are generally conducted under consistent operating conditions without any changes in the whole life cycle test. However, the monitored tribological systems are usually operating in a complex environment with different states such as wind turbine gearbox and vehicle gearbox in industry $[4,7,28]$. Ma et al. $[29,31]$ proposed a numerically efficient algorithm and strategy for the monitoring of time-varying and multimode characteristics on 
industrial processes. This dynamic adaptive fusion method is suitable for current electrostatic monitoring features and needs to be investigated to offer a possible solution for electrostatic monitoring technique.

In this work, the strategy is applied and developed for electrostatic monitoring of tribo-contacts. The electrostatic monitoring principle, experimental setup, and initial electrostatic features are introduced in Section 2. The advanced dynamic adaptive fusion method combined with standardized electrostatic characteristics is presented in Section 3. Test results of rolling bearings and further application in railcar gearbox are analyzed and discussed in Section 4. Finally, conclusions are made in Section 5.

\section{Electrostatic Monitoring Method of Tribo- Contacts}

2.1. Principle of Electrostatic Monitoring. Electrostatic monitoring technique is a complex process from the initial charge generation in tribological system to the final acquisition and analysis of electrostatic signals, as seen in Figure 1. Based on the previous investigations, five causes (i.e., tribo-charging, tribo-emission, tribo-electrification, surface charging, and debris charging) were thought to be the possible charge generation mechanisms in tribo-contacts $[8,26,32]$. In general, the electrostatic sensor (ES) consists of five parts: inner inductive probe with sensing face, middle insulation layer, outside shielding layer, signal output end, and installation part. When a charge source (e.g., charged debris or lubricant charge species) passes through the front of the sensor, a charge response can be detected by the usual inductive processes. With suitable signal processing methods, the extracted electrostatic features can be used to reflect the wear condition of tribo-contacts in mechanical system and detect early faults.

2.2. Experimental Setup. Based on the principle of electrostatic monitoring, life cycle experiments with two electrostatic sensors (ESs) and monitoring system are conducted on the test rig of rolling bearings, as shown in Figure 2. Four bearings are installed in the test chamber monitored by ESs and driven by a motor. The rotation speed and radial load of rolling bearings can be set and adjusted in the experiment. The test rig itself has a temperature and vibration monitoring system which can order a warning to reflect the operating condition if the rolling bearing runs into severe failure. Electrostatic signals are collected by the monitoring system.

2.3. Electrostatic Feature Extraction. First, the collected original electrostatic signals are a series of time-domain values with initial denoising by a simple charge filter. Then, due to the high sampling frequency and longtime duration of monitoring resulted with big data, signals are processed with feature extraction method to reflect the electrostatics level intuitively and effectively. According to the previous investigations $[13,24]$, the conventional extracted features most used in time domain to detect abnormal in electrostatic signals are root mean square parameter (RMS), peak-peak parameter (PP), impulse factor (IF), and clearance factor (CLF), etc., as listed in Table 1.x $(n)$ is a signal series for $n=1$, $2, \ldots, N$ and $N$ is the number of data points.

Although these extracted electrostatic features are seemed to be effective under certain stable operation conditions, problems arose when it comes to time-varying operation conditions. The electrostatic signal can be influenced by the change of operation conditions (e.g., rotation speed, load, and torque etc.), especially under the real industrial environment. Thus, a novel algorithm called moving window local outlier factor (MWLOF) is introduced here in order to solve the problem. The method is based on a data mining method called local outlier factor (LOF) algorithm and used in industrial monitoring processes, which further adapts the changes of operating modes by adding a fast-moving window scheme $[29,30]$. The process of the algorithm combined with electrostatic monitoring signals as a dynamic adaptive fusion method is presented in the following section.

\section{Dynamic Adaptive Method of Electrostatic Monitoring}

3.1. Algorithm of LOF. In the algorithm of LOF according to equation (1), all the $k$ nearest neighbors of $x_{a}(a=1,2$, $\ldots, N)$ should be found out for a set $X=\left[x_{1}, x_{2}, \ldots\right.$, $\left.x_{N}\right] \in R^{D \times N}$ of training data, while the neighborhood set of $x_{a}$ is then denoted as $\operatorname{knn}\left(x_{a}\right)$.

$$
d\left(x_{a}, x_{b}\right)=\sqrt{\sum_{n=1}^{D}\left|x_{a n}-x_{b n}\right|^{2}(a \neq b)} .
$$

The $\mathrm{k}$-distance neighborhood of $x_{a}$, i.e., the minimum $k-$ th of $d\left(x_{a}, x_{b}\right)$ and $\left(a \neq b, x_{b} \in \operatorname{knn}\left(x_{a}\right)\right)$, is calculated by the Euclidean distance of $k$ - th closest value from $x_{a}$ and denoted as $k$ - distance $\left(x_{a}\right)$. The reachability distance of $x_{a}$ relative to $x_{b}$ can be recognized as

$$
\text { reach-dist }\left(x_{a}, x_{b}\right)=\max \left\{k-\operatorname{distance}\left(x_{b}\right), d\left(x_{a}, x_{b}\right)\right\} \text {. }
$$

The local reachability density (LRD) of $x_{a}$ can be calculated through the equation:

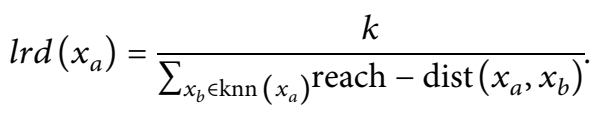

The LOF of $x_{a}$ is computed from the LRD of $x_{a}$ and nearest neighbors through:

$$
\operatorname{lof}\left(x_{a}\right)=\frac{1}{k} \cdot \sum_{x_{b} \in \operatorname{knn}\left(x_{a}\right)} \frac{\operatorname{lrd}\left(x_{b}\right)}{\operatorname{lrd}\left(x_{a}\right)} .
$$

From the definition of LOF, it indicates that the larger $\operatorname{lof}\left(x_{a}\right)$ is, the more likely $x_{a}$ is an outlier. When $x_{a}$ is not, the value of $\operatorname{lof}\left(x_{a}\right)$ is close to 1 ; while $x_{i}$ is an outlier, the value of $\operatorname{lof}\left(x_{a}\right)$ will distinctly larger than 1 . It is worth to mention that the value of LOF equals to 1 is an ideal condition which cannot be applied to determine whether these data are abnormal or not. 

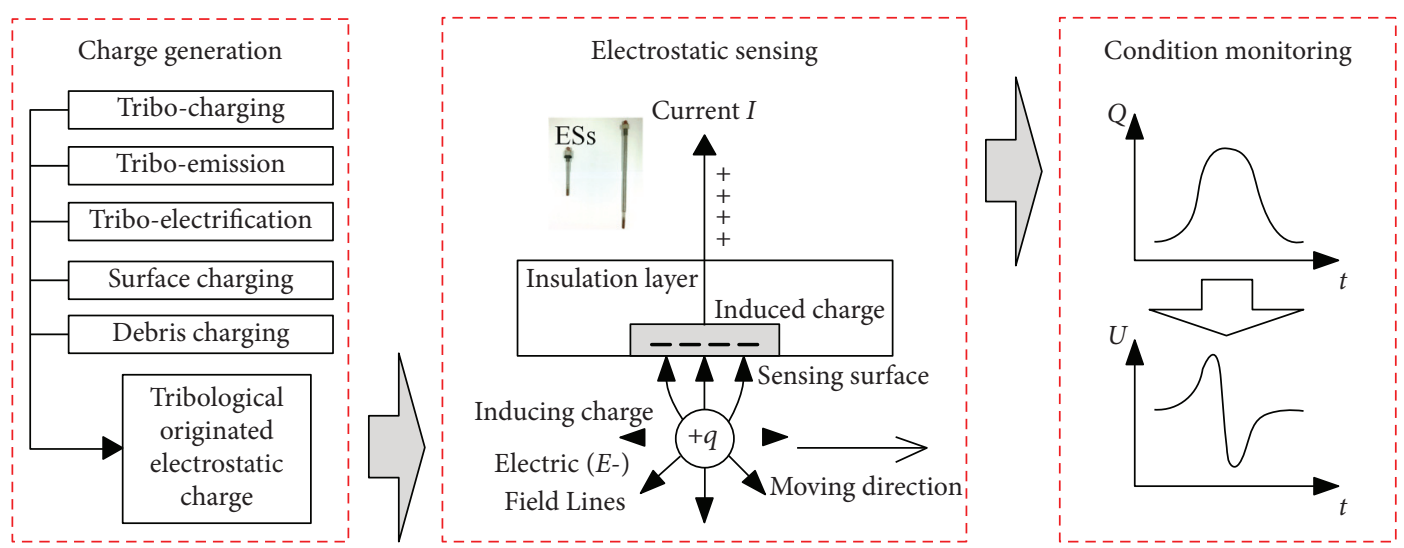

FIGURE 1: A schematic diagram of the electrostatic monitoring principle.

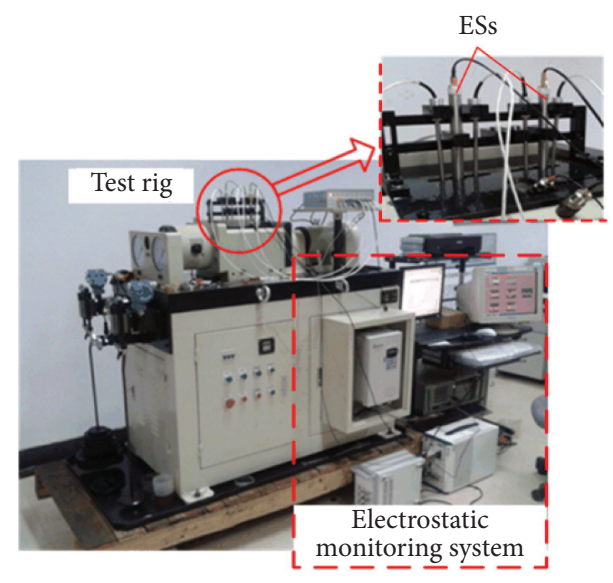

(a)

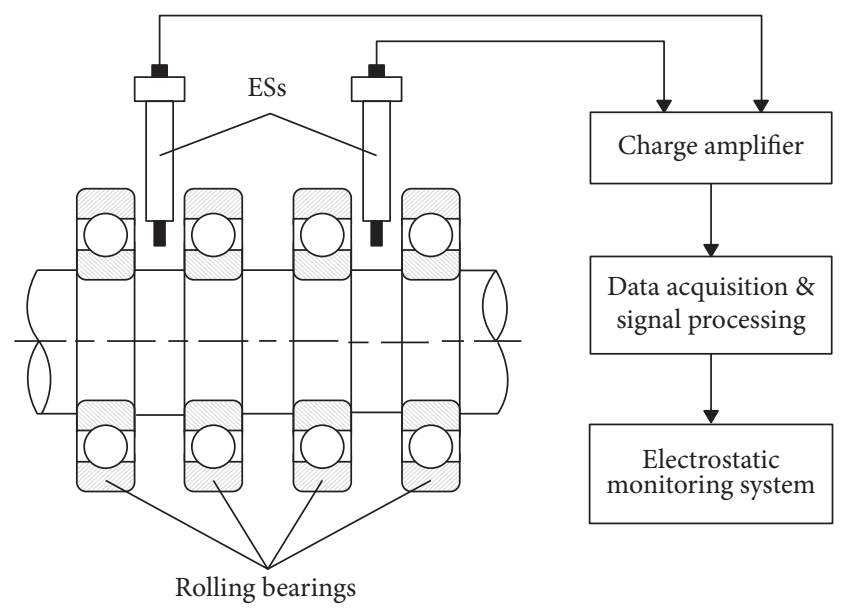

(b)

FIGURE 2: Experimental setup of electrostatic monitoring on rolling bearing. (a) Test rig; (b) schematic of the structure.

3.2. Algorithm of MWLOF. To calculate the value of LOF and make an initialization for each sample $x_{j}(j=1,2, \ldots, L)$ with a dimension $D$ in the old window $W_{1}$ by the distance according to equation (1), all the $k$ closest $x_{j}$ marked as $\mathrm{knn}_{1}\left(x_{j}\right)$ are found. Then, with the incorporated $k$-distance ${ }_{1}\left(x_{j}\right)$, the reachability distance of $x_{j}$ relative to $x_{p}\left(p \neq j, x_{p} \in \operatorname{knn}_{1}\left(x_{j}\right)\right)$ denoted as reach $-\operatorname{dist}_{1}\left(x_{j}, x_{p}\right)$ is calculated. Then the value of LRD $\operatorname{lr} d_{1}\left(x_{j}\right)$ and the value of LOF $l o f_{1}\left(x_{j}\right)$ are computed according to equations (3) and (4) sequentially.

There are three matrices in Figure 3 to process online updating of the data: original old window $W_{i}$, current new window $W_{i+1}$, and middle window $\widetilde{W}$ with some samples removed. For a window size $L$, the details of the two-step adaption procedure are demonstrated as follows.

\section{Step 1. abandon}

The result of elements (mean, variance, and covariance) for the middle window by removing the oldest sample can be calculated by equations (5) to (8). By moving $x_{i}$ away, the whole neighborhood relationship is updated from $W_{i}$ to $\widetilde{W}$, while a set $S_{i-1}$ to store the samples is constructed and satisfied the criterion below equation (9). Where $\mathrm{knn}_{i}\left(x_{j}\right)$ is the set of neighborhood $x_{j}$ in $W_{i}$. Then the values of $k$ - distance for the samples in $S_{i-1}$ will turn into new values equation (10).

$$
\begin{aligned}
\tilde{\mu}= & \frac{1}{L-1}\left(L \mu_{i}-x_{i}\right) \\
\Delta \widetilde{\mu}= & \mu_{i}-\widetilde{\mu} \\
\widetilde{\sigma}(m)^{2}= & \frac{L-1}{L-2}\left[\sigma_{i}(m)\right]^{2}-\frac{L-1}{L-2}[\Delta \widetilde{\mu}(m)]^{2} \\
& -\frac{\left[x_{i}(m)-\mu_{i}(m)\right]^{2}}{L-2}, \quad m=1,2, \ldots, D \\
\sum= & \operatorname{diag}[\sigma(1), \sigma(2), \ldots, \sigma(D)] \\
S_{i-1}= & S_{i-1} \cup\left\{x_{j}\right\}, \text { if } i \neq j, x_{j} \in W_{i} \\
& \text { and } x_{i} \in \operatorname{knn}_{i}\left(x_{j}\right),
\end{aligned}
$$

$k$ - distance $_{i}\left(x_{j}\right)=(k+1)-$ distance $_{i}\left(x_{j}\right)$,

$$
\text { if } x_{j} \in S_{i-1} \text {. }
$$

Step 2. addition

New data will be added in the matrix of the window after $x_{i+L}$ is considered normal, and these elements (mean, 
TABLE 1: Conventional electrostatic time-domain features.

\begin{tabular}{lr}
\hline Features & Formula \\
\hline Mean value $x_{M}$ & $x_{M}=\frac{\sum_{n=1}^{N} x(n)}{N}$ \\
Root mean square $x_{\mathrm{RMS}}$ & $x_{\mathrm{RMS}}=\sqrt{\frac{\sum_{n=1}^{N}(x(n))^{2}}{N}}$ \\
Standard deviation $x_{\mathrm{STD}}$ & $x_{\mathrm{STD}}=\sqrt{\frac{\sum_{n=1}^{N}\left(x(n)-x_{\mathrm{M}}\right)^{2}}{N-1}}$ \\
Peak-peak $x_{\mathrm{PP}}$ & $x_{\mathrm{PP}}=\max \left(x_{n}\right)-\min \left(x_{n}\right)$ \\
Skewness $x_{\mathrm{SKE}}$ & $x_{\mathrm{SKE}}=\frac{\sum_{n=1}^{N}\left(x(n)-x_{\mathrm{M}}\right)^{3}}{(N-1) x_{\mathrm{ST}}^{3}}$ \\
Kurtosis $x_{\mathrm{KUR}}$ & $x_{\mathrm{KUR}}=\frac{\sum_{n=1}^{N}\left(x(n)-x_{\mathrm{M}}\right)^{4}}{(N-1) x_{\mathrm{STD}}^{4}}$ \\
Peak factor $x_{\mathrm{PF}}$ & $x_{\mathrm{PF}}=\frac{\max |x(n)|}{x_{\mathrm{RMS}}}$ \\
Impulse factor $x_{\mathrm{IF}}$ & $x_{\mathrm{IF}}=\frac{\max |x(n)|}{\sum_{n-1}^{N}|x(n)| / N}$ \\
Clearance factor $x_{\mathrm{CLF}}$ & $x_{\mathrm{CLF}}=\frac{m_{\max |x(n)|}}{\left(\sum_{n-1}^{N}|x(n)| / N\right)^{2}}$ \\
\hline
\end{tabular}

$$
\begin{aligned}
& \text { Old Window } \quad \text { Intermediate Data New Window } \\
& W_{t}=\left[\begin{array}{c}
x_{i} \\
x_{i+1} \\
\vdots \\
x_{i+L-1}
\end{array}\right]_{L \times D} \quad \Longrightarrow \tilde{W}=\left[\begin{array}{c}
x_{i+1} \\
\vdots \\
x_{i+L-1}
\end{array}\right]_{(L-1) \times D} \quad \square W_{i+1}=\left[\begin{array}{c}
x_{i+1} \\
\vdots \\
x_{i+L-1} \\
x_{i+L}
\end{array}\right]_{L \times D} \\
& \left(\mu_{i}, \sum_{i}, k \text {-distance } \text { dit }_{i}, \quad\left(\tilde{\mu}_{i}, \tilde{\Sigma}_{i}, k \text {-distance }, S_{i-1}\right) \quad\left(\mu_{i+1}, \sum_{i+1}, k \text {-distance }{ }_{i+1}\right. \text {, }\right. \\
& \text { reach-dist } \left.\left.{ }_{i}, \operatorname{lrd}_{i}, \operatorname{lof}_{i}\right) \quad \text { reach-disd } d_{i+1}, \operatorname{lrd}_{i_{+1}}, \operatorname{lof}_{i_{+1}}\right)
\end{aligned}
$$

FIgURE 3: Two-step adaptation procedure of the MWLOF strategy.

variance, and covariance) will also be updated through equations (11) to (14). However, the values of $k$-distance for those samples will also be updated according to equation (15). Where $x_{j} \in \widetilde{W}$ and then the set $S_{i-1}$ will be augmented as equation (16). The reachability distance at this step will be recalculated if its nearest samples in $W_{i+1}$ belongs to $S_{i-1}$ according to equation (17). The value of LRD and LOF in the new window $W_{i+1}$ can be calculated by updating the distance through equations (18) and (19).

$$
\begin{aligned}
\mu_{i+1} & =\frac{1}{L}\left[(L-1) \widetilde{\mu}+x_{i+L}\right], \\
\Delta \mu_{i+1} & =\mu_{i+1}-\widetilde{\mu}, \\
\sigma_{i+1}(m)^{2} & =\frac{L-2}{L-1}[\tilde{\sigma}(m)]^{2}+\left[\Delta \mu_{i+1}(m)\right]^{2}+\frac{\left[x_{i+L}(m)-\mu_{i+1}(m)\right]^{2}}{L-1}, \quad m=1,2, \ldots, D, \\
\sum_{i+1} & =\operatorname{diag}\left[\sigma_{i+1}(1), \sigma_{i+1}(2), \ldots, \sigma_{i+1}(D)\right], \\
k-\text { distance }_{i+1}\left(x_{j}\right) & = \begin{cases}(k-1)-\operatorname{distance}\left(x_{j}\right), & \text { if } x_{i+L} \text { is among } k-1 \text { nearest neighbors of } x_{j}, \\
d\left(x_{i+L}, x_{j}\right), & \text { if } x_{i+L} \text { is the } k \text { th }- \text { nearest neighbor of } x_{j}, \\
k-\operatorname{distance}\left(x_{j}\right), & \text { otherwise, } \\
S_{i-1} & =S_{i-1} \cup\left\{x_{j}\right\}, \text { if } x_{j} \notin S_{i-1} \text { and } x_{i+L} \in \mathrm{knn}_{i+1}\left(x_{j}\right),\end{cases}
\end{aligned}
$$




$$
\begin{aligned}
& \text { reach }-\operatorname{dist}_{i+1}\left(x_{j}, x_{p}\right)= \begin{cases}k-\operatorname{distance}_{i+1}\left(x_{p}\right), & \max \left\{d\left(x_{j}, x_{p}\right)\right\} \text { if } x_{p} \in S_{i-1}, \\
\text { reach }-\operatorname{dist}_{i}\left(x_{j}, x_{p}\right), & \text { otherwise, }\end{cases} \\
& \text { where } p \neq j, x_{j} \in W_{i+1} \text { and } x_{p} \in \operatorname{knn}_{i+1}\left(x_{j}\right) \text {. }
\end{aligned}
$$

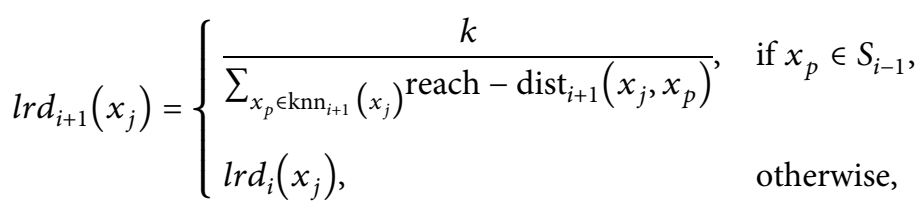

$$
\begin{aligned}
& \text { where } p \neq j, x_{j} \in W_{i+1} \text { and } x_{p} \in \operatorname{knn}_{i+1}\left(x_{j}\right) \text {. } \\
& \operatorname{lof}_{i+1}\left(x_{j}\right)= \begin{cases}\frac{1}{k} \cdot \sum_{x_{p} \in \mathrm{knn}_{i+1}\left(x_{j}\right)} \frac{\operatorname{lrd} d_{i+1}\left(x_{p}\right)}{\operatorname{lrd} d_{i+1}\left(x_{j}\right)}, & \text { if } x_{p} \in S_{i-1}, \\
\operatorname{lof}_{i}\left(x_{j}\right), & \text { otherwise, }\end{cases}
\end{aligned}
$$

\subsection{MWLOF-Based Electrostatic Monitoring of Dynamic} Adaptive Process. Considering the actual outputs of the electrostatic monitoring signals are a series of data with many parameters of different magnitude features collected by multiple sensors; therefore, it is necessary to standardize the training data before applying the LOF algorithm. Otherwise, the variables with large order of magnitude will occupy a larger weight when calculating the distance, and when the fault happens in the variables with small order of magnitude, the sign of fault will be difficult to be accurately characterized. The specific steps of standardization method are as follows.

First, assuming that the total number of electrostatic sensor probes used in the monitoring system, especially a single sensor may have multiple poles and output terminals, is $d$ and equals to the total number of electrostatic signal output terminals. The output signal corresponding to the time series $n$ of each sensor probe during monitoring is $A^{j}=\left\{a_{1}^{j}, a_{2}^{j}, \ldots, a_{n}^{j}\right\}, \quad(j=1,2, \ldots, d)$. The feature extraction method in Section 2.3 is used to process each electrostatic signals in $A^{j}$, getting multiple characteristic features on the same time series and forming the corresponding initial sample data set $B^{j}=\left\{b_{1}, b_{2}, \ldots, b_{m}\right\} \in R^{N \times m}$. Where $N$ represents the number of samples on the time series of the feature parameters extracted from the converted signal and $m$ represents the number of feature parameters extracted from the converted signal, i.e., the number of variables.

Then, the data set $B^{j}$ should be standardized to let each value in the uniform scale with the mean value of 0 and the variance of 1 through

$$
X_{i}^{j}=\frac{b_{i}-\operatorname{mean}\left(b_{i}\right)}{\sigma\left(b_{i}\right)}, \quad i=1,2, \ldots, m,
$$

where $b_{i}$ presents a variable value of $B^{j}, X_{i}^{j}$ is the result of standardization, mean (.) presents the mean of value, and $\sigma($.) presents the standard deviation of the value. Then it forms a new data set $X=\left\{X^{1}, X^{2}, \ldots, X^{d}\right\}=\left\{x_{1}, x_{2}, \ldots\right.$,
$\left.x_{D}\right\} \in R^{N \times D}$, where $x_{i} \in R^{N}$ and $N$ is the number of samples, $X^{j} \in R^{N \times m},(j=1,2, \ldots, d)$ present the data set of each electrostatic output signal that is transformed and standardized. $D$ is the total number of variable values in the new sample set and it can be calculated as $D=m \times d$. The new formed data set $X$ is the input of adaptive monitoring process with the MWLOF algorithm. Figure 4 shows the flow diagram of the proposed electrostatic signal based MWLOF algorithm and the adaptive process monitoring method.

The offline initialization steps are as follows: at first, $L$ samples of the electrostatic signals are collected under the current operating condition to form the initial old window $W_{1}$. An offline model is established according to equations (1) to (4) on the basics of the standardized electrostatic samples. According to the initial window $W_{1}$, by specifying a confidence level $(1-\alpha) \%$ and applying kernel density estimation (KDE), a control limit for the electrostatic values of LOF is built to estimate following experimental data.

Then, the online model updating and monitoring steps through the switch rule and the two-step adaption approach are summarized in following seven steps: (a) standardize the new electrostatic sample $x_{i+L}$ with $\mu_{i}$ and $\sum_{i}$ of the window $W_{i}$. (b) Calculate the distance between $x_{i+L}$ and $x_{i+1}, x_{i+2}, \ldots, x_{i+L-1}$ with $l o f_{i}\left(x_{i+L}\right)$ from the window $W_{i}$. (c) The flag value will be switched from 0 to 1 by an operator to accept new samples as normal if the mode changes, and each new data will be accepted as then directly skip to step (e); The flag will be reset to 0 automatically until termination of updating is reached. Otherwise, it will move to the next step when the value of flag is 0 . (d) $x_{i+L}$ will be regarded as an outlier and cannot be added into the window if the value is above the threshold of the window $W_{i}$, i.e., $\operatorname{lo} f_{i}\left(x_{i+L}\right)>\operatorname{limit}-\operatorname{lo} f_{i}$, and the cycle continues for the next new sample. If not, it will move to the next step. (e) Update the model by inserting $x_{i+L}$ to take place of $x_{i}$ through equations (5) to (19). (f) The threshold will be recalculated through KDE when $l o f_{i}\left(x_{i+L}\right)>1$. (g) The alarm will be triggered at the 


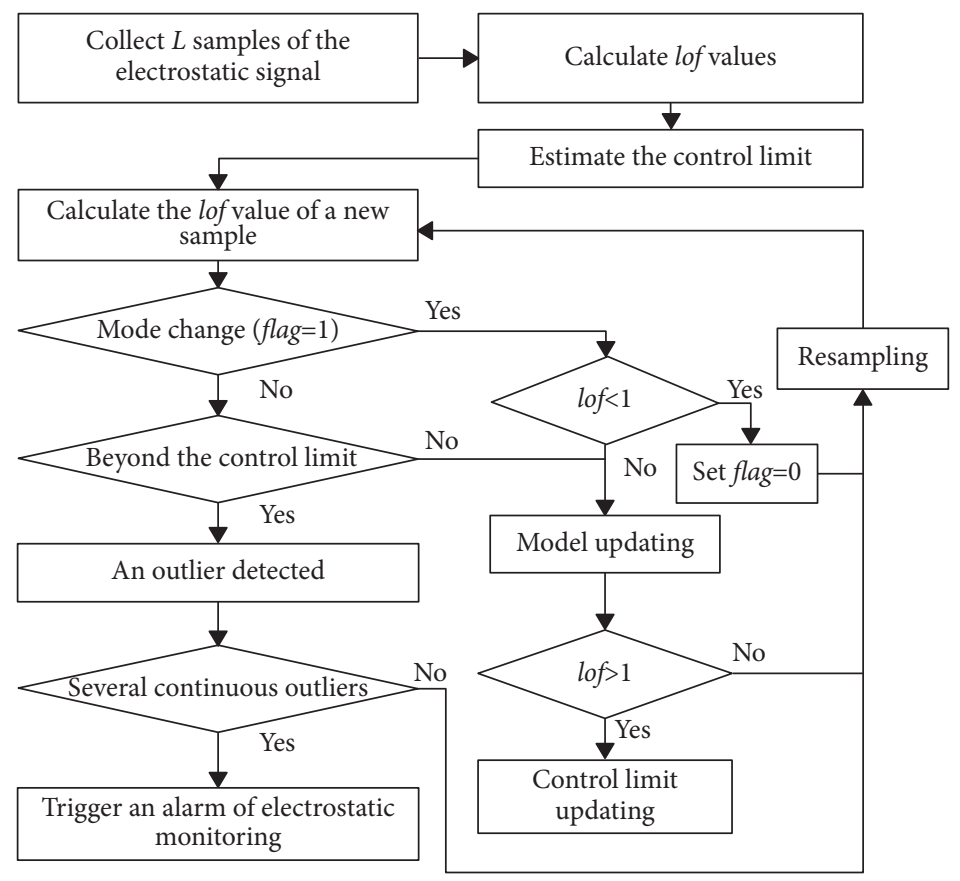

FIgURE 4: Flow diagram of dynamic adaptive electrostatic monitoring process based on MWLOF.

final step when several consecutive outliers are detected. It is worth mention that, according to the previous investigations $[29,30]$, the parameters $k$ and window size are set to 30 and 800 , respectively, which are suitable for the model while applying in the following experimental analysis.

\section{Experimental Results and Analysis}

4.1. Experiment of the Rolling Bearing Test. In the experiment of rolling bearing with the test rig introduced in Section 2 (Figure 2(a)), a life cycle test with two operating stages is conducted with electrostatic monitoring method. At the beginning with first stage, it runs stable under $2000 \mathrm{r} / \mathrm{min}$ rotation speed and $20 \mathrm{kN}$ radial load. After 800 samples, it switches to the second stable stage, with rotation speed and radial load are both increased, under $3000 \mathrm{r} / \mathrm{min}$ and $30 \mathrm{kN}$. Two tests are conducted in the experiment with good rolling bearings. In the first test, it keeps running to the failure with the total number of 2290 monitoring samples, as shown in Figure 5. Figures 5(a)5(c), respectively, show the detailed results by using MWLOF with mode change method, MWLOF without mode change at 800 sample, and with basic LOF method. Figure 5(d) shows the result with some traditional features. Figure 5(e) shows the inner race fault detected of rolling bearing after the test.

From Figure 5(a), it can be seen that the value of electrostatic lof keeps steady at a low level and is below the corresponding threshold before early fault occurs (about 1900th sample), which reflects the normal running condition of rolling bearing at this period. At about 800th sample, the value of electrostatic lof has a relatively high pulse corresponding to the operation of stage switches, which lasts a few moment/samples then fall to the normal level. By using the MWLOF algorithm with mode change, the high pulses, caused by the change of operating stages without any obvious fault of rolling bearing, are also regarded as normal signal to be calculated and below new threshold. It shows that the rolling bearing of the test in the whole life cycle before early fault occurs, i.e., from 1 st to 1911th sample in this experiment, runs well without any abnormality. After 1911th sample, some values of electrostatic lof above the threshold began to appear frequently, which indicate the early degradation of rolling bearing performance that began to show up. After 2099th sample, some continuous abnormal values began to appear corresponding to the further degradation of rolling bearing performance. After 2156th sample, the value of electrostatic lof began to keep above the threshold and rising until far larger than the threshold, which reflects the severe wear and failure of rolling bearing, as shown in Figure 5(e). From the whole test, it can be seen that, with the dynamic adaptive fusion method of MWLOF algorithm considering mode change, the result of electrostatic monitoring consists with the degradation of rolling bearing performance from the initial normal operating condition to occurrence of early fault and final failure at the end.

Comparing Figures 5(b) and 5(a), it can be seen that in the whole test by using electrostatic monitoring of MWLOF algorithm without mode switches, the moment of multiple (1911th sample), continuous (2099th sample), and consistent (2156th sample) abnormal values above the threshold occurrence corresponding to early, further degradation and severe fault of rolling bearing are the same with the method with mode change. However, the part of stage change (after 800th sample) with a series of high values above the threshold is misconsidered as the occurrence of early fault. Meanwhile, owing to the update of mode without values in the mode change, some values of electrostatic lof in the region between 1200th and 1800th above the threshold are also misconsidered as abnormal. It proves the advantage of MWLOF algorithm with mode change in the electrostatic monitoring of rolling bearing test. 

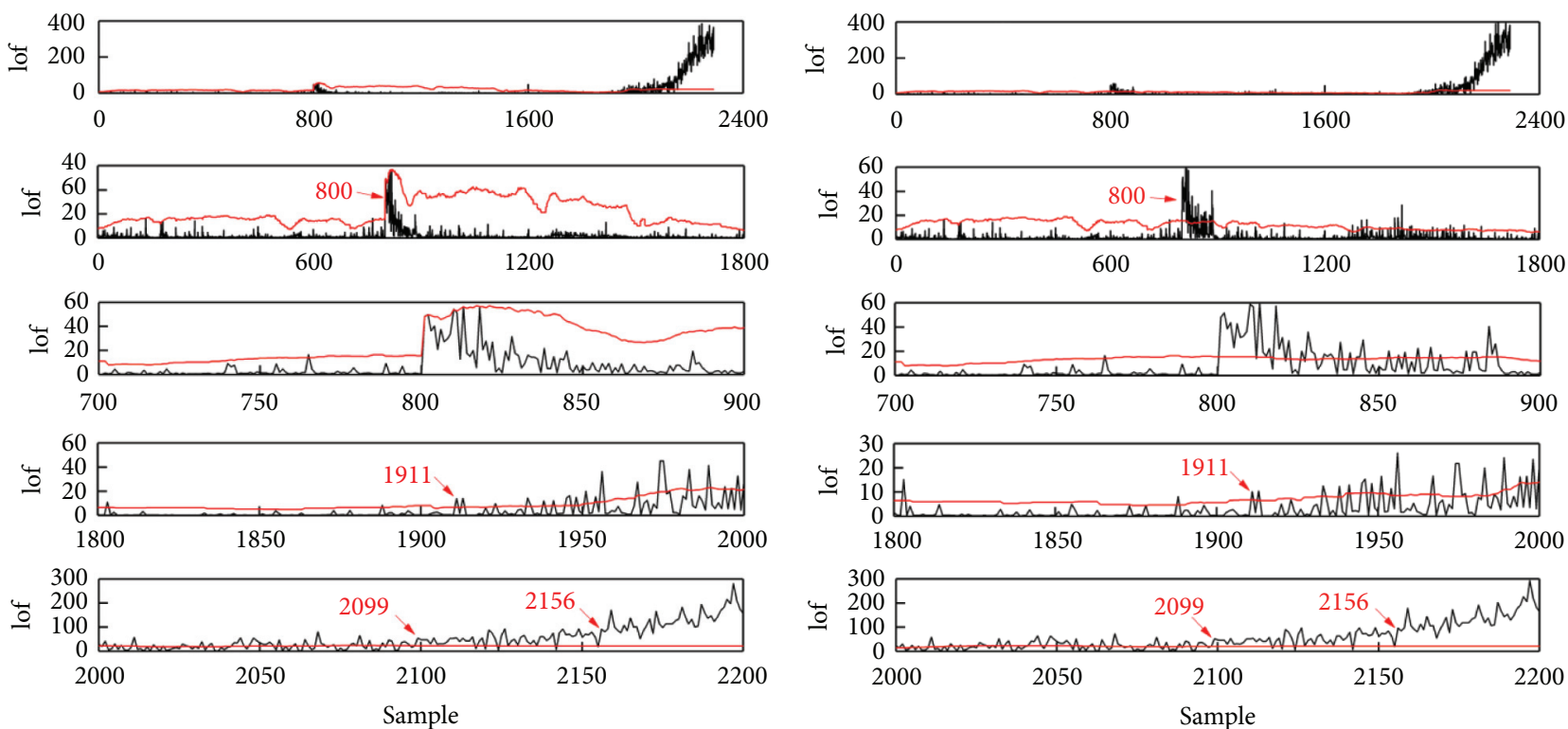

- lof

- lof

_ threshold

(b)
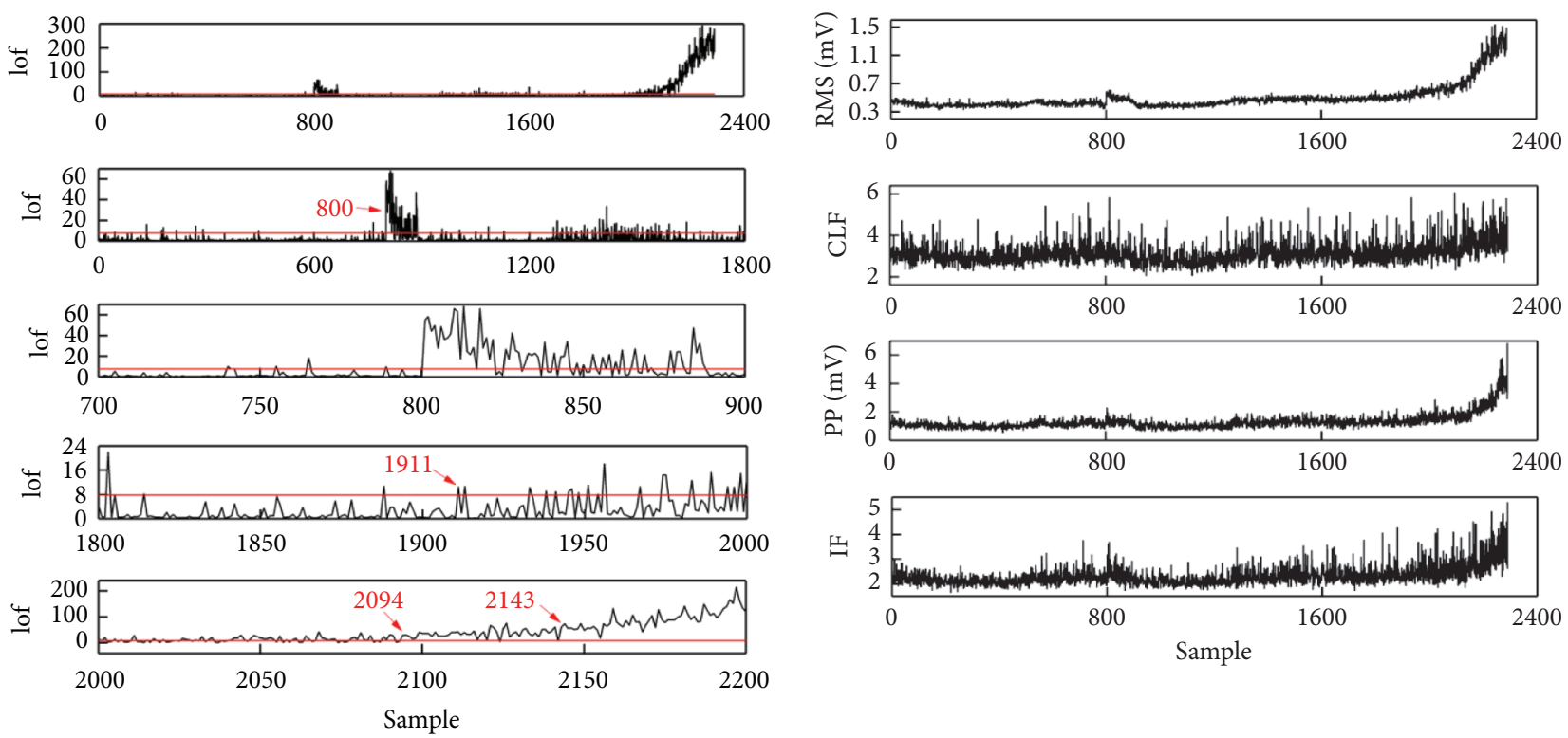

— lof

- threshold

(c)

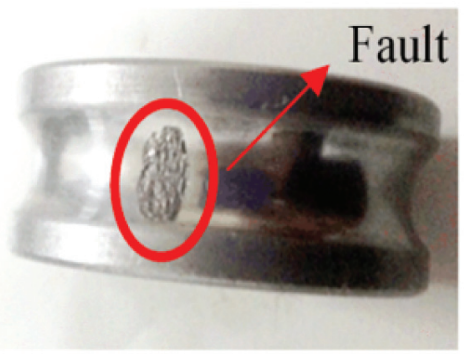

(e) (d)

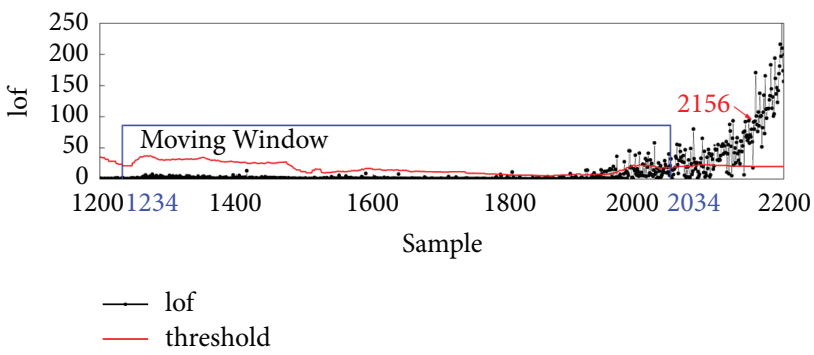

(f)

FIGURE 5: Electrostatic monitoring results of rolling bearing first test. (a) MWLOF with mode change; (b) MWLOF without mode change; (c) basic LOF; (d) traditional features; (e) inner race fault; (f) abnormal points reach " $2 \sigma$ " standard. 
Comparing Figures 5(c) to 5(a) and 5(b), it can be seen that electrostatic monitoring result of LOF algorithm without moving window is different from MWLOF monitoring algorithm. Owing to the static threshold without considering any update, the 1911th sample is not the first signal to start the appearance of frequent abnormal values, and the first moment of continuous and consistent abnormal values above the threshold occurrence is forward to 2094th and 2143th samples. Meanwhile, similar to the monitoring result of MWLOF without mode change, the part of stage change (after 800th sample) with a series of high values above the threshold is also misconsidered as the occurrence of early fault. The total amount of abnormal values considered as false alarm under normal operating conditions before 1911th with three algorithms are calculated with a result of 257 samples (MWLOF with mode change), 332 samples (MWLOF without mode change), and 426 samples (LOF) after the test. It indicates that the MWLOF algorithm with mode change is a better method for electrostatic monitoring than the other two methods. It is also worth mentioning that the LOF algorithm with a static threshold determined by the chosen training data will easily influence the result of monitoring, i.e., low threshold with too many abnormal values and high threshold without detection of early fault degradation, which is hard to be applied in electrostatic monitoring of rolling bearing.

From Figures 5(d), it can be seen that in the whole test, the electrostatic representative dimensioned features, i.e., RMS and PP, have a good trend to reflect the degradation and also with some high pulses at the moment of stage change, while the electrostatic representative dimensionless features, i.e., CLF and IF, have a slight trend to reflect the degradation and without any obvious pulses at the moment of stage change. So, it is hard to evaluate the condition of rolling bearing with only one of the extracted features and is also hard to give the threshold suit for every situation considering the variations of electrostatic level in different monitoring system. It is necessary and a good solution for electrostatic signals to have a fusion combining all the time-domain features.

Although MWLOF algorithm can dynamically adjust the threshold height with the change of bearing operating conditions, it is difficult to completely define the bearing fault failure by a single scale standard. Therefore, it is also necessary to quantitatively divide the threshold standard of bearing fault by different scales. From the perspective of probability distribution, we can set the length scale for the upper limit of the sample of bearing anomaly. In the sample detection of bearings, the sample data of fixed length generally conform to the law of Gaussian distribution. Therefore, it can be based on Pauta criterion to determine the upper limit of abnormal samples. If the random variable $x$ follows a Gaussian distribution of mathematical expectation $(\mu)$ and standard variance $\left(\sigma^{2}\right)$, it is recorded as

$$
x \sim N\left(\mu, \sigma^{2}\right) \text {. }
$$

Then, the probability density function is expressed as

$$
f(x) \frac{1}{\sigma \sqrt{2 \pi}} e^{-(x-u)^{2} / 2 \sigma^{2}}
$$

The expected value $(\mu)$ of Gaussian distribution determines its position, while the standard deviation $(\sigma)$ determines its amplitude.

In practical application, the area of a certain interval on the horizontal axis under the normal curve reflects the percentage of the number of cases in this interval to the total number of cases, or the probability that the variable value falls in this interval. The sample length of MWLOF moving iteration is 800 continuous sampling points, and the specific distribution of normal points in the " $3 \sigma$ " horizontal axis interval is shown in Table 2.

Due to the limited experimental error and data amount, we adopt the " $2 \sigma$ " standard to evaluate bearing fault, that is, if 36 sample points in 800 consecutive samples exceed the threshold, judge the bearing fault and send out early warning alarm; if 36 consecutive sample points exceed the threshold, the equipment will enter the emergency shutdown state and wait for the maintenance personnel to check and deal with it.

Figure 5(f) shows the partial enlargement of the moving window when it reaches the " $2 \sigma$ " theoretical fault of the inner ring. In the 800 samples' interval of (1234 to 2034)th, the number of normal samples is 764 , accounting for $95.5 \%$ of the total number of samples, while the number of abnormal samples exceeding the threshold reaches 36 , which is beyond the standard range of " $2 \sigma$," so the bearing fault is judged and given early warning at point 2034th. After the 2156th sample, 36 consecutive samples exceeded the threshold, so the equipment shall be shut down for processing. A judgment criterion combining the threshold height and the length of " $2 \sigma$ " sample interval can quantitatively evaluate the health status of bearings and provide an accurate judgment basis for maintenance personnel.

One more test is conducted to further verify the algorithms and experiment under the same procedure, i.e., start with $2000 \mathrm{r} / \mathrm{min}$ rotation speed and $20 \mathrm{kN}$ radial load at stable stage one and then switches to the second stable stage with $3000 \mathrm{r} / \mathrm{min}$ rotation speed and $30 \mathrm{kN}$ radial load, with new rolling bearings replaced. In the second test, it keeps running to the failure with the total number of 2170 monitoring samples, as shown in Figure 6. Figures 6(a)-6(c), respectively, show the detailed results by using MWLOF with mode change method, MWLOF without mode change at 800 sample, and with basic LOF method. Figure 6(d) shows the result with some traditional features. Figure 6(e) shows the outer race fault detected of rolling bearing after the test.

Comparing Figures 6(a) and 5(a), the electrostatic monitoring results of rolling bearing in these two tests by using MWLOF algorithm with mode change are similar. The value of electrostatic lof keeps stable in a low level and is below the corresponding threshold before early fault occurs (about 1850th sample), which reflects the normal running condition of rolling bearing at this period. At about 800th sample, the value of electrostatic lof has a relatively high pulse corresponding to the operation of stage switches, which lasts a few moment/samples then falls to the normal level. By using the MWLOF algorithm with mode change, the high pulses, caused by the change of operating stages without any obvious fault of rolling bearing, are also 
TABLE 2: Samples distribution in the " $3 \sigma$ " interval of the moving window.

\begin{tabular}{lcc}
\hline Interval & Distribution probability (\%) & Abnormal amount \\
\hline$(\mu-\sigma, \mu+\sigma)$ & 68.27 & 254 \\
$(\mu-2 \sigma, \mu+2 \sigma)$ & 95.45 & 36 \\
$(\mu-3 \sigma, \mu+3 \sigma)$ & 99.73 & 2 \\
\hline
\end{tabular}

regarded as normal signal to be calculated and below new threshold. It shows that the rolling bearing of the test in the whole life cycle before early fault occurs, i.e., from 1st to 1872th sample in this experiment, runs well without any abnormal. After 1872th sample, some values of electrostatic lof above the threshold began to appear frequently, which indicate the early degradation of rolling bearing performance began to show up. After 1988th sample, some continuous abnormal values began to appear corresponding to the further degradation of rolling bearing performance. After 2032th sample, the value of electrostatic lof began to keep above the threshold and rising until far larger than the threshold, which reflects the severe wear and failure of rolling bearing, as shown in Figure 6(e). From the whole second test, it can also be seen that, with the dynamic adaptive fusion method of MWLOF algorithm considering mode change, the result of electrostatic monitoring consists with the degradation of rolling bearing performance from the initial normal operating condition to occurrence of early fault and final failure at the end.

Comparing Figures 6(b) and 6(a), it can also be seen that in the whole second test by using electrostatic monitoring of MWLOF algorithm without mode switches, the moment of multiple (1872th sample), continuous (1988th sample), and consistent (2032th sample) abnormal values above the threshold occurrence corresponding to early, further degradation and severe fault of rolling bearing are the same with the method with mode change. However, the part of stage change (after 800th sample) with a series of high values above the threshold is misconsidered as the occurrence of early fault. Meanwhile, owing to the update of mode without values in the mode change, some values of electrostatic lof in the region between 1200th and 1800th above the threshold are also misconsidered as abnormal. It again proves the advantage of MWLOF algorithm with mode change in the electrostatic monitoring of rolling bearing test.

Comparing Figures 6(c) to 6(a) and 6(b), the electrostatic monitoring result of LOF algorithm without moving window is also different from MWLOF monitoring algorithm in the second test. Although the 1872th sample is still the first signal to start the appearance of frequent abnormal values, the first moment of continuous and consistent abnormal values above the threshold occurrence is backward to 2007th and 2042th sample. Meanwhile, similar to the monitoring result of MWLOF without mode change, the part of stage change (after 800th sample) with a series of high values above the threshold is also misconsidered as the occurrence of early fault. The total amount of abnormal values considered as false alarm under normal operating conditions before 1911th with three algorithms is calculated with a result of 233 samples (MWLOF with mode change), 310 samples (MWLOF without mode change), and 412 samples (LOF) after the test. It again indicates that the MWLOF algorithm with mode change is a better method for electrostatic monitoring than the other two methods. It is also worth to mention that, owing to the original training data, the first test has a relatively low threshold to forward the trend of degradation, while the second test has a relatively high static threshold to backward the trend.

From Figure 6(d), it can also be seen that in the whole test the electrostatic representative dimensioned features, i.e., RMS and PP, have a good trend to reflect the degradation and also with some high pulses at the moment of stage change, while the electrostatic representative dimensionless features, i.e., CLF and IF, have a slight trend to reflect the degradation and without any obvious pulses at the moment of stage change.

Figure 6(f) is a partial enlargement of the moving window when it reaches the " $2 \sigma$ " theoretical fault of the outer ring. In the 800 samples interval of (1158 to 1958)th, the number of normal samples is 764 and the number of abnormal samples reaches 36 , exceeding " $2 \sigma$ " interval range. At 1958th sample, a bearing failure is determined and an early warning is issued. After the 2156th sample, there are 36 consecutive samples that exceed the threshold, and the equipment needs to be shut down for processing.

In summary, the experiment proves the effectiveness of MWLOF algorithm with mode change applied on the electrostatic rolling bearing monitoring with various operating conditions in the whole life cycle test.

4.2. Application of the Railcar Gearbox Test. In order to certify the suitability of the method in real application, a further experiment of electrostatic monitoring on a gearbox used in railcar is conducted in the load fatigue test, as shown in Figure 7. A pair of gears and rolling bearings are monitored by electrostatic sensors. The whole procedure of the test is also shown in Table 3. It has four stages, and both the rotation and torque have two directions. Each stage lasts 40 hours and thus the whole test has 160 hours. In the first stage of the test (0 to 40 hours), the gearbox keeps operating under $1641 \mathrm{r} / \mathrm{min}$ rotation speed and $2078 \mathrm{~N} \cdot \mathrm{m}$ torque both at one direction. When the test enters the second stage (41 to 80 hours), the rotation turns to another direction with the same value of $1641 \mathrm{r} / \mathrm{min}$ while keeps the torque constant without any changes. When it enters the third stage ( 81 to 120 hours) after the second stage finished, the torque turns to another direction with the same value of $2078 \mathrm{~N} \cdot \mathrm{m}$ while keeps the rotation without any changes. When it enters the fourth stage (121 to 160 hours), the rotation again turns to another direction with the same value of $1641 \mathrm{r} / \mathrm{min}$ while keeps the torque constant without any changes. It is worth mention that there is only a stop between the second and third stages, while there are no stops between the first and second and third and fourth stages throughout the whole test. The result of electrostatic monitoring with MWLOF algorithm with mode change for the whole test is shown in Figure 8.

From Figure 8(a), it can be seen that most values of electrostatic lof keep under the threshold in the whole test. 

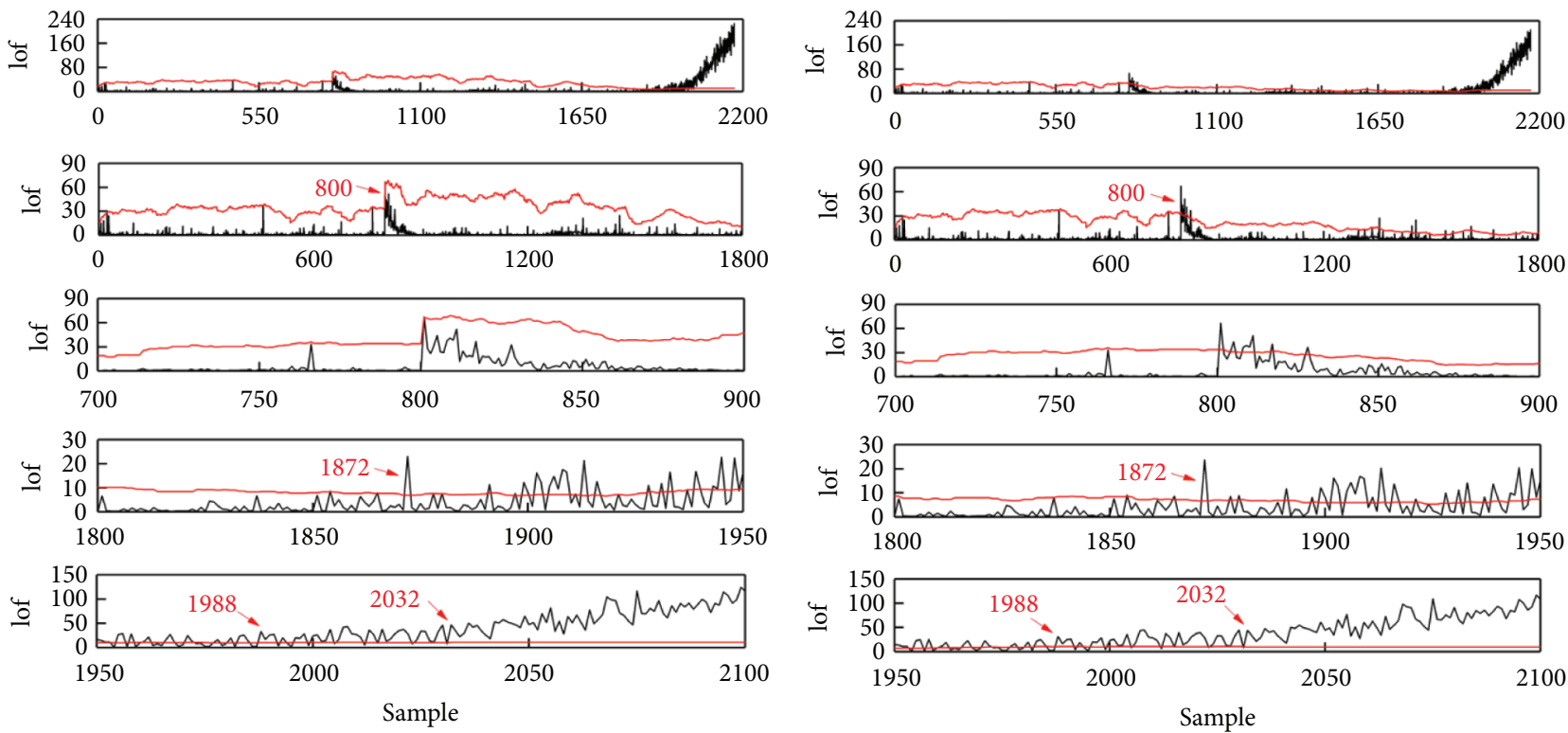

- lof

— threshold

$\begin{array}{ll}\text { — } & \text { lof } \\ \text { threshold }\end{array}$

(a)
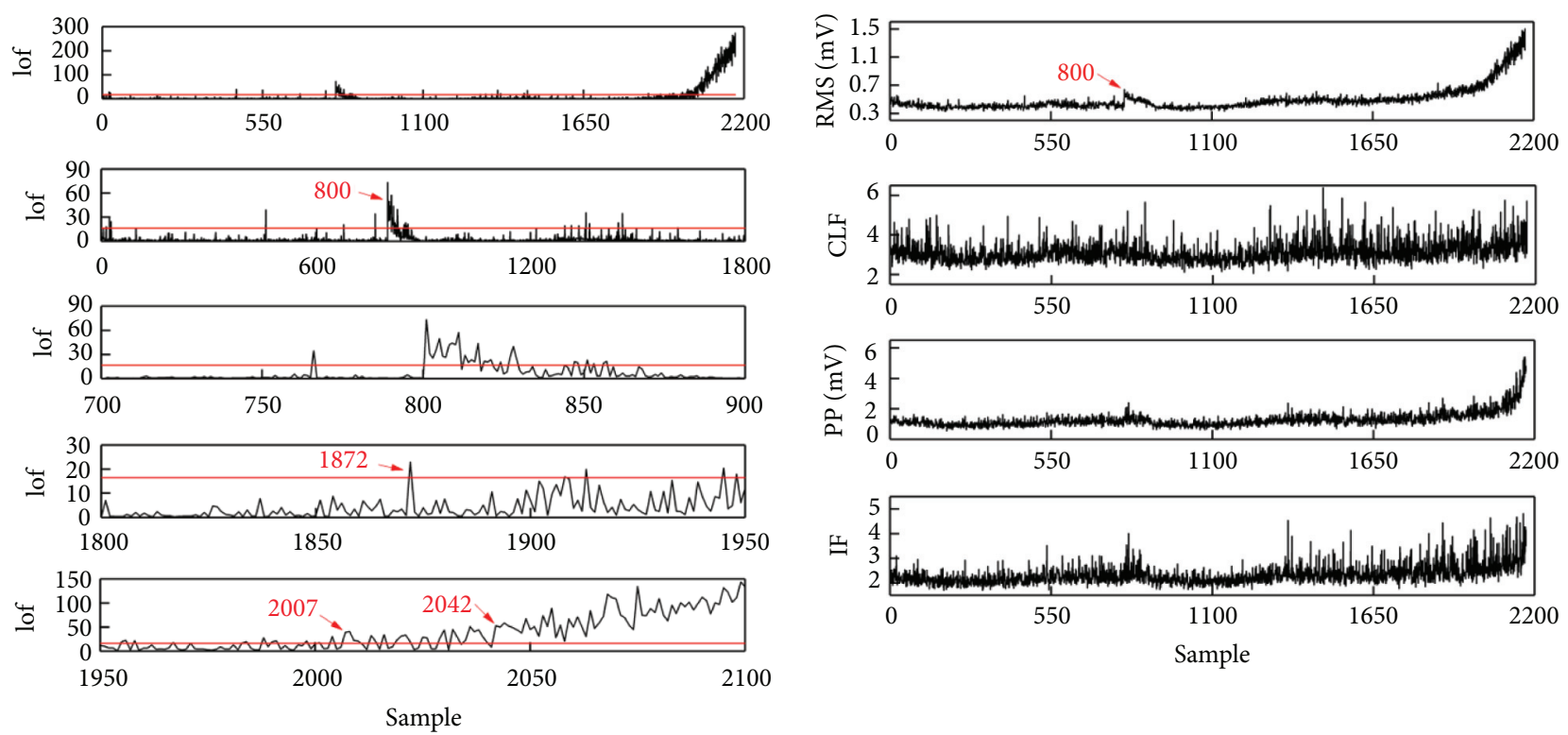

- lof

— threshold

(c)

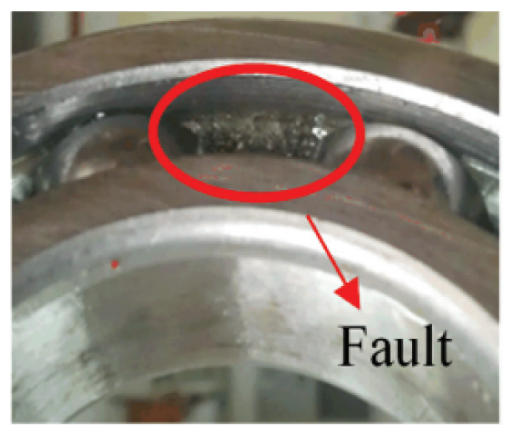

(d)

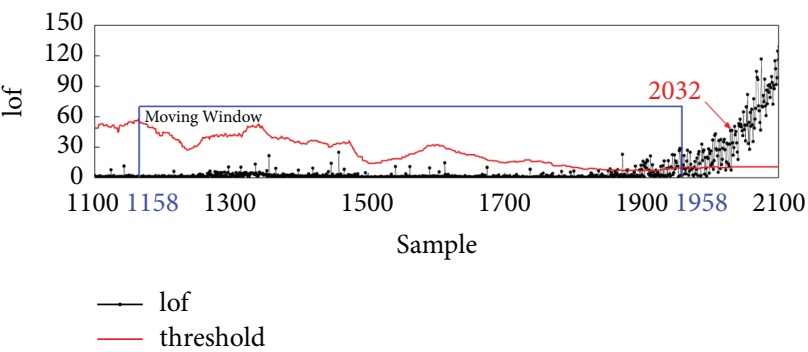

(f)

FIGURE 6: Electrostatic monitoring results of rolling bearing second test. (a) MWLOF with mode change; (b) MWLOF without mode change; (c) basic LOF; (d) traditional features; (e) outer race fault; (f) abnormal points reach " $2 \sigma$ " standard. 

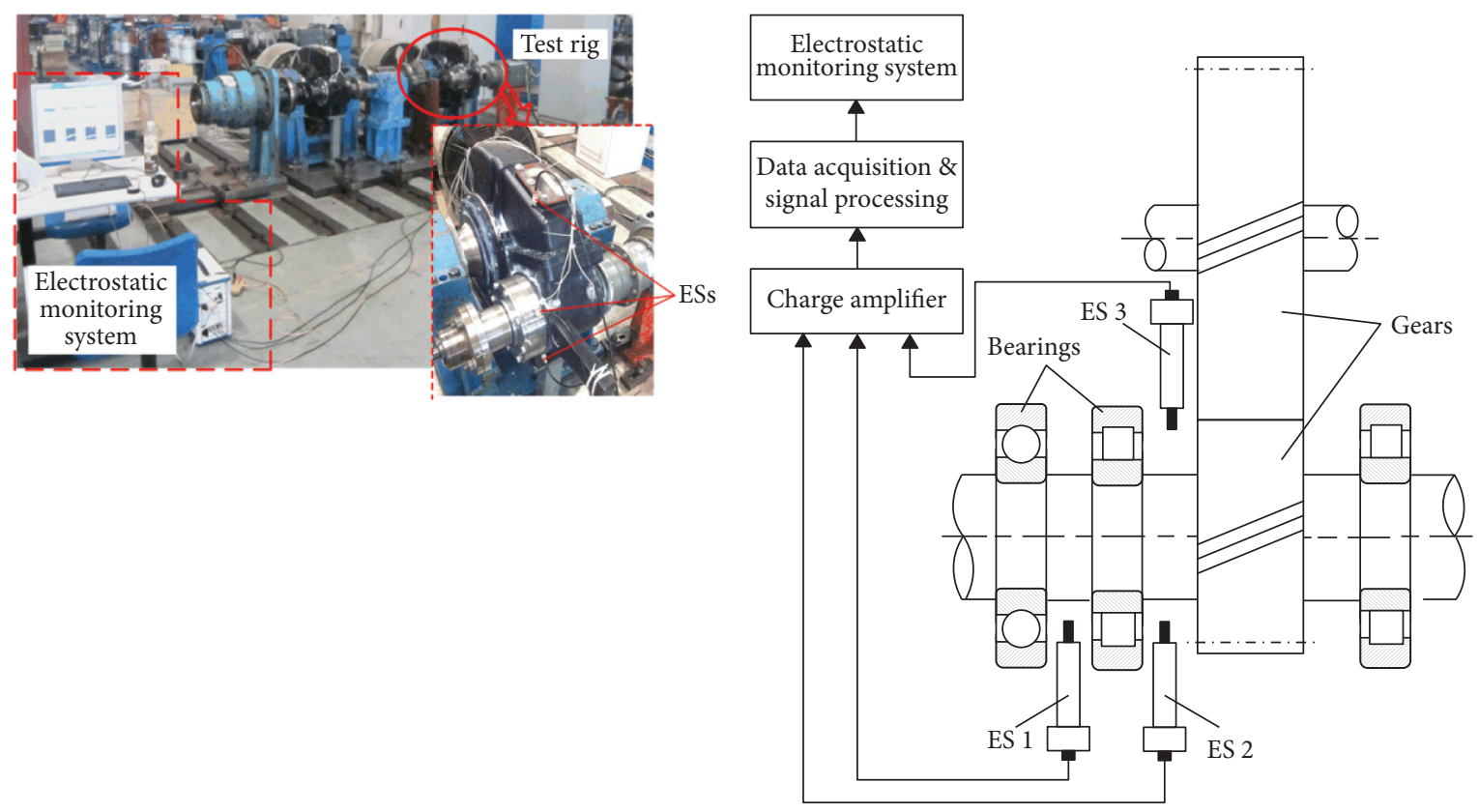

(a)

(b)

FIgURE 7: Experimental setup of electrostatic monitoring on railcar gearbox. (a) Test rig; (b) schematic of the structure.

TABLE 3: Operating condition of the gearbox load fatigue test.

\begin{tabular}{lccc}
\hline Stage & Rotation speed $(\mathrm{r} / \mathrm{min})$ & Torque $(\mathrm{N} \cdot \mathrm{m})$ & Duration $(\mathrm{h})$ \\
\hline 1 & 1641 & 2078 & 40 \\
2 & -1641 & 2078 & 40 \\
3 & -1641 & -2078 & 40 \\
4 & 1641 & -2078 & 40 \\
\hline
\end{tabular}
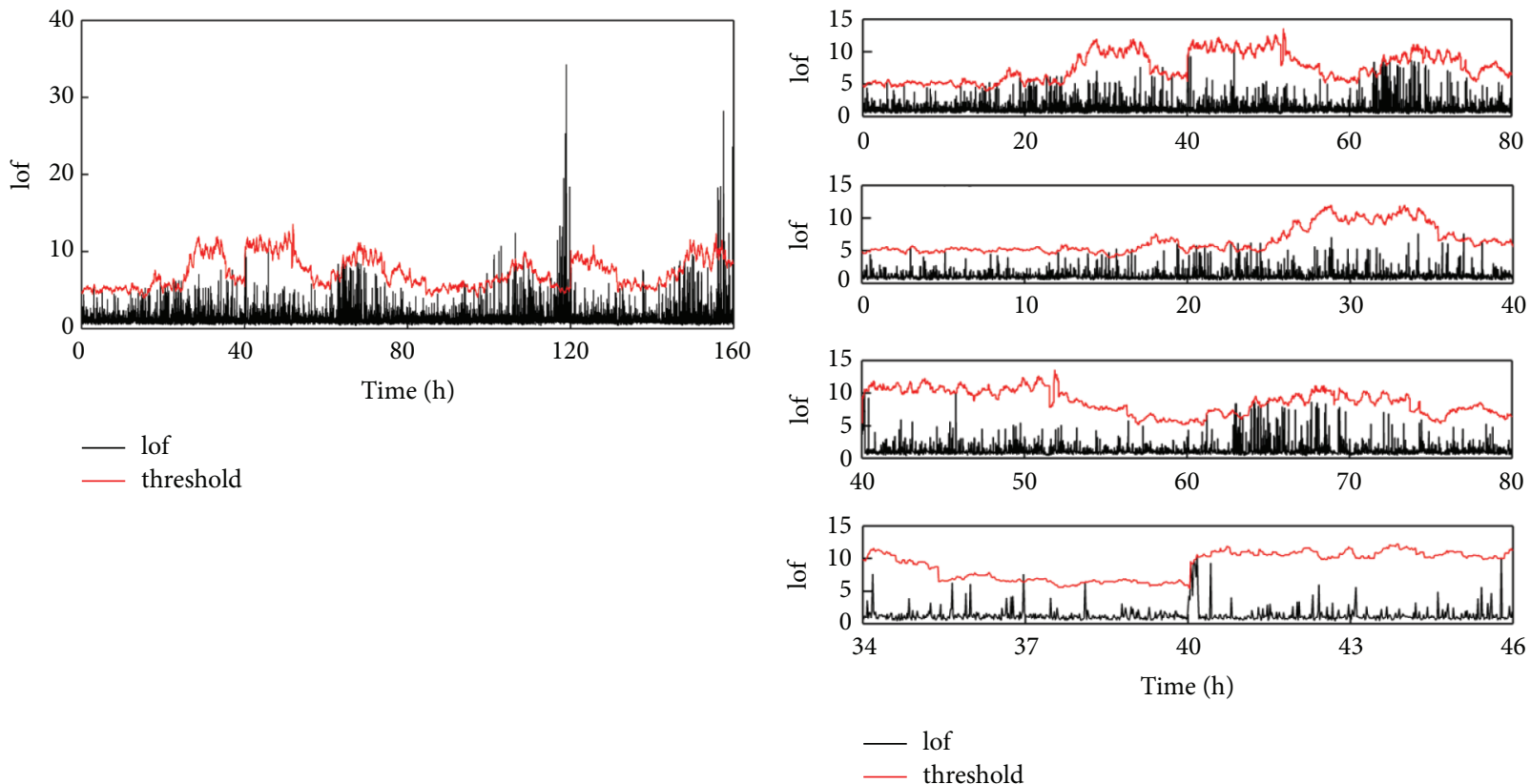

(a)

(b)

Figure 8: Continued. 

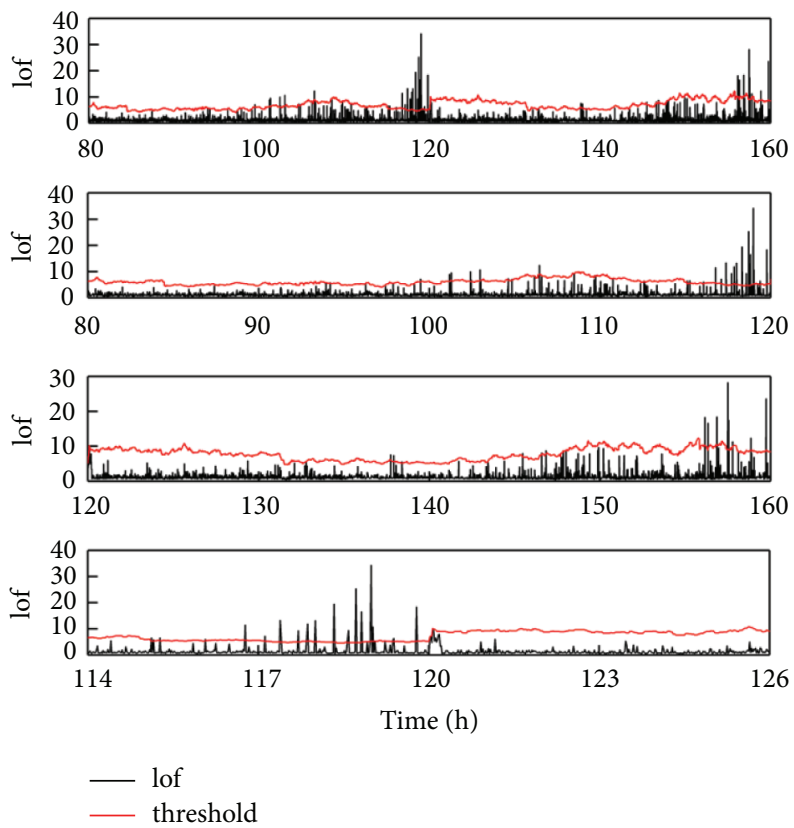

(c)

FIGURE 8: Electrostatic monitoring of railcar gearbox: (a) total result; (b) detailed results of first half; (c) detailed results of second half.

Figures $8(\mathrm{~b})$ and $8(\mathrm{c})$ are the detailed results of the monitoring signals in first two stages and last two stages, respectively. From Figure 8(b), it can be seen that the value of electrostatic lof has a relatively high pulse after 40 hours corresponding to the transform of operating stages (1st to 2nd), which lasts a period then falls to the normal level. By using the MWLOF algorithm with mode change, the high pulses caused by the change of operating stages are regarded as normal signal to be calculated and below new threshold. The same situation occurs at the transform of operating stages (3rd to 4th) after 120 hours, seen in Figure 8(c). It can also be seen that there are obvious early degradations of the gearbox monitored by electrostatic sensors at the end of both third stage (115 to 120 hours) and fourth stage (156 to 160 hours) with some high pulses above the threshold, corresponding to the two rotation directions. It indicates that the degradation mainly occurs at the gear transmission part rather than the bearing part. An unpacking inspection after the test shows that there are slight pitting and scuffing in the surface of gear at both sides corresponding to the early degradation, while there are no server wear faults that lead to a failure for gear and bearings corresponding to the stable monitoring results of vibration and temperature. Similarly, quantitative analysis of the data is carried out from the perspective of probability distribution. We divide the interval from the change of working conditions and collect 2400 sample points under each working condition. Table 4 shows the number of abnormal points and sample normality rate in each stage. After actual sampling, we found that the normal rate of samples under the working conditions of the four stages remained above $95.45 \%$, that is, within the " $2 \sigma$ " range of all samples in each stage. From this, it is verified that there is no obvious fault in the gearbox despite the abnormal
TABle 4: Samples situation of the gearbox.

\begin{tabular}{lcc}
\hline State & Normal rate (\%) & Abnormal amount \\
\hline 1 & 99.58 & 10 \\
2 & 99.71 & 7 \\
3 & 98.33 & 40 \\
4 & 99.42 & 14 \\
\hline
\end{tabular}

value of static electricity in the monitoring. The result of the investigation proves the effectiveness of electrostatic monitoring with dynamic adaptive fusion method, i.e., MWLOF algorithm with mode change, to apply in industrial wear components.

\section{Conclusions}

Electrostatic monitoring is a potential technique for the PHM of tribological system in industry. However, it is difficult to integrate various features from multiple electrostatic sensors and overcome the influence of operating condition changes for the system. This paper considered the characteristics of electrostatic signals and introduced a modified MWLOF algorithm with mode change as a dynamic adaptive fusion method to indicate the performance and verified with experiments of rolling bearings and gearbox.

This investigation resulted in the following observations: parts of traditional electrostatic time-domain features (mainly have dimension) have a good correlation with degradation while are easily influenced by the change of operating conditions, whereas other features (mainly are dimensionless) are opposite; the MWLOF algorithm has a value of lof as an integrated indicator with a dynamic 
adaptive threshold through the moving window based on the standardized and unified electrostatic features from the outputs of multiple electrostatic sensors; and the mode change module added in the process is to follow the switches of operating conditions based on MWLOF algorithm. In the two tests of rolling bearings, the monitoring result of electrostatic lof by using MWLOF algorithm with mode change has a good correlation with the performance of degradation without the misdiagnosed at the part of operating condition switches. Early degradation is detected with frequent abnormal values, and the consistent high values above the threshold reflect the severe fault of wear in the whole life test. The result of MWLOF algorithm with mode change is better than MWLOF without mode change and basic LOF algorithm. In the further test of railcar gearbox including gears and bearings, the proposed modified dynamic adaptive fusion method proves its feasibility on electrostatic monitoring of tribo-contacts in industry.

\section{Data Availability}

The data used to support the findings of this study are available from the corresponding author upon request.

\section{Conflicts of Interest}

The authors declare no conflicts of interest.

\section{Acknowledgments}

The authors would like to thank Prof. Michael G. Pecht for his support to the leading author's study at University of Maryland, College Park. The authors would like to thank Dr. Yang Ou for his support at Maryland. This research was supported by the National Natural Science Foundation of China, under grant nos. 51705221 and 51805229, and Jiangsu Government Scholarship for Overseas Studies.

\section{References}

[1] D. Rengasamy, M. Jafari, B. Rothwell, X. Chen, and G. P. Figueredo, "Deep learning with dynamically weighted loss function for sensor-based prognostics and health management," Sensors, vol. 20, no. 3, p. 723, 2020.

[2] K.-L. Tsui, N. Chen, Q. Zhou, Y. Hai, and W. Wang, "Prognostics and health management: a review on data driven approaches," Mathematical Problems in Engineering, vol. 2015, Article ID 793161, 17 pages, 2015.

[3] G. Li, Y. Wang, J. He, T. Hou, L. Du, and Z. Hou, "Fault forecasting of a machining center tool magazine based on health assessment," Mathematical Problems in Engineering, vol. 2020, Article ID 5796965, 10 pages, 2020.

[4] P. Tchakoua, R. Wamkeue, M. Ouhrouche, F. Slaoui-Hasnaoui, T. Tameghe, and G. Ekemb, "Wind turbine condition monitoring: state-of-the-art review, new trends, and future challenges," Energies, vol. 7, no. 4, pp. 2595-2630, 2014.

[5] X. Li, J. Cai, J. Cai et al., "Joint optimization of sampling interval and control for condition-based maintenance using availability maximization criterion," Journal of Systems Engineering and Electronics, vol. 29, no. 1, pp. 203-215, 2018.
[6] X. Ye, C. Dong, and T. Liu, “A review of machine vision-based structural health monitoring: methodologies and applications," Journal of Sensors, vol. 2016, Article ID 7103039, 10 pages, 2016.

[7] M. Hossain, A. Abu-Siada, and S. Muyeen, "Methods for advanced wind turbine condition monitoring and early diagnosis: a literature review," Energies, vol. 11, no. 5, p. 1309, 2018.

[8] L. Wang, R. Wood, and T. Harvey, "Electrostatic charge-a novel condition monitoring technology," Condition Monitor, vol. 248, pp. 5-9, 2007.

[9] Z. Wen, J. Hou, and J. Atkin, "A review of electrostatic monitoring technology: The state of the art and future research directions," Progress in Aerospace Sciences, vol. 94, pp. 1-11, 2017.

[10] L. Li, H. Hu, and K. Tang, "A specially-designed electrostatic sensor for the condition monitoring of rolling bearings," Measurement Science and Technology, vol. 32, pp. 1-14, 2021.

[11] Y Zhang, A Wang, and H Zuo, "Roller bearing performance degradation assessment based on fusion of multiple features of electrostatic sensors," Sensors (Basel, Switzerland), vol. 19, pp. 2-15, 2019.

[12] J. E. Booth, K. D. Nelson, T. J. Harvey et al., "The feasibility of using electrostatic monitoring to identify diesel lubricant additives and soot contamination interactions by factorial analysis," Tribology International, vol. 39 , no. 12, pp. 1564-1575, 2006.

[13] R. Liu, H. Zuo, J. Sun, and L. Wang, "Electrostatic monitoring of wind turbine gearbox on oil-lubricated system," Proceedings of the Institution of Mechanical Engineers, Part C: Journal of Mechanical Engineering Science, vol. 231, no. 19, pp. 3649-3664, 2017.

[14] J. Sun, F. Wang, and S. Ning, "Aircraft air conditioning system health state estimation and prediction for predictive maintenance," Chinese Journal of Aeronautics, vol. 33, no. 3, pp. 947-955, 2020.

[15] S. J. Wright, D. W. Dixon-Hardy, and P. J. Heggs, "Aircraft air conditioning heat exchangers and atmospheric fouling," Thermal Science and Engineering Progress, vol. 7, pp. 184-202, 2018.

[16] P. Liu, H. Zuo, and J. Sun, "The electrostatic sensor applied to the online monitoring experiments of combustor carbon deposition fault in aero-engine," IEEE Sensors Journal, vol. 14, no. 3, pp. 686-694, 2013.

[17] Y. Zhang and A. wang, "Remaining useful life prediction of rolling bearings using electrostatic monitoring based on twostage information fusion stochastic filtering," Mathematical Problems in Engineering, vol. 2020, Article ID 2153235, 12 pages, 2020.

[18] T. Harvey, R Wood, and H. Powrie, "Electrostatic wear monitoring of rolling element bearings," Wear, vol. 263, no. 7-12, pp. 1492-1501, 2007.

[19] J. Booth, T. Harvey, and R. Wood, "Scuffing detection of TU3 cam-follower contacts by electrostatic charge condition monitoring," Tribology International, vol. 43, no. 1-2, pp. 113-128, 2010.

[20] T. J. Harvey, R. J. K. Wood, G. Denuault, and H. E. G. Powrie, "Effect of oil quality on electrostatic charge generation and transport," Journal of electrostatics, vol. 55, no. 1, pp. 1-23, 2002.

[21] H. Mao, H. Zuo, and H. Wang, "Debris recognition methods in the lubrication system with electrostatic sensors," Mathematical Problems in Engineering, vol. 2018, Article ID 8043526, 15 pages, 2018. 
[22] J. Sun, R. Wood, and L. Wang, "Wear monitoring of bearing steel using electrostatic and acoustic emission techniques," Wear, vol. 259, no. 7-12, pp. 1482-1489, 2005.

[23] M. Craig, T. Harvey, and R. Wood, "Advanced condition monitoring of tapered roller bearings, part 1," Tribology International, vol. 42, no. 11-12, pp. 1846-1856, 2009.

[24] Y. Zhang, H. Zuo, and F. Bai, "Feature extraction for rolling bearing fault diagnosis by electrostatic monitoring sensors," Proceedings of the Institution of Mechanical Engineers, Part C: Journal of Mechanical Engineering Science, vol. 229, no. 10, pp. 1887-1903, 2015.

[25] H. Mao, H. Zuo, and H. Wang, "Electrostatic sensor application for on-line monitoring of wind turbine gearboxes," Sensors, vol. 18, no. 10, p. 3574, 2018.

[26] R. Liu and H. Zuo, "Mathematical model, simulation, and experimental calibration of electrostatic wear-site sensor," IEEE Sensors Journal, vol. 17, no. 8, pp. 2428-2438, 2017.

[27] R. C. Liu, H. F. Zuo, J. Z. Sun, and L. Wang, "Simulation of electrostatic oil line sensing and validation using experimental results," Tribology International, vol. 105, pp. 15-26, 2017.

[28] J. Wang, J. Yang, and Q. Li, "Quasi-static analysis of the nonlinear behavior of a railway vehicle gear system considering time-varying and stochastic excitation," Nonlinear Dynamics, vol. 93, no. 2, pp. 463-485, 2018.

[29] Y. Ma, H. Shi, H. Ma, and M. Wang, "Dynamic process monitoring using adaptive local outlier factor," Chemometrics and Intelligent Laboratory Systems, vol. 127, pp. 89-101, 2013.

[30] Y. Ma, H. Shi, and M. Wang, "Adaptive local outlier probability for dynamic process monitoring," Chinese Journal of Chemical Engineering, vol. 22, no. 7, pp. 820-827, 2014.

[31] H. Zhang, Y. Zhang, Z. Sun, Y. Hu, and G. Wang, "Design and research of electrostatic sensor based on aero-engine airway electrostatic monitoring technology," Journal of Physics: Conference Series, vol. 1237, no. 5, pp. 1-7, 2019.

[32] Z. Wen, J. Hou, and Z. Jiang, "Formation mechanism analysis and detection of charged particles in an aero-engine gas path," International Journal of Aeronautical and Space Sciences, vol. 16, no. 2, pp. 247-253, 2015. 